\title{
National and sub-national age-sex specific and cause-specific mortality and disability-adjusted life years (DALYs) attributable to household air pollution from solid cookfuel use (HAP) in Iran, 1990-2013
}

\author{
Mehrnoosh Abtahi $^{\mathrm{a}}$, Ali Koolivand ${ }^{\mathrm{b}}$, Sina Dobaradaran ${ }^{\mathrm{c}, \mathrm{d}, \mathrm{e}}$, Kamyar Yaghmaeian ${ }^{\mathrm{f}}$, \\ Anoushiravan Mohseni-Bandpei ${ }^{\mathrm{a}}$, Shokooh Sadat Khaloo ${ }^{\mathrm{g}}$, Sahand Jorfi, ${ }^{\mathrm{h}, \mathrm{i}}$, Reza Saeedi ${ }^{\mathrm{j}}$, \\ ${ }^{a}$ Department of Environmental Health Engineering, School of Public Health, Shahid Beheshti University of Medical Sciences, Tehran, Iran \\ b Department of Environmental Health Engineering, Faculty of Health, Arak University of Medical Sciences, Arak, Iran \\ c The Persian Gulf Marine Biotechnology Research Center, Bushehr University of Medical Sciences, Bushehr, Iran \\ d Department of Environmental Health Engineering, Faculty of Health, Bushehr University of Medical Sciences, Bushehr, Iran \\ e Systems Environmental Health, Oil, Gas and Energy Research Center, Bushehr University of Medical Sciences, Bushehr, Iran \\ ${ }^{\mathrm{f}}$ Department of Environmental Health Engineering, School of Public Health and Institute for Environmental Research, Tehran University of Medical Sciences, Tehran, Iran \\ ${ }^{g}$ School of Health, Safety and Environment, Shahid Beheshti University of Medical Sciences, P.O. Box 16858-116, Tehran, Iran \\ h Environmental Technology Research Center, Ahvaz Jundishapur University of Medical Sciences, Ahvaz, Iran \\ ${ }^{i}$ Department of Environmental Health Engineering, School of Public Health, Ahvaz Jundishapur University of Medical Sciences, Ahvaz, Iran \\ j Department of Health Sciences, School of Health, Safety and Environment, Shahid Beheshti University of Medical Sciences, Tehran, Iran
}

\section{A R T I C L E I N F O}

\section{Keywords:}

Air pollution

Solid fuel use

Health effects

Spatiotemporal trend

Iran

\begin{abstract}
A B S T R A C T
National and sub-national mortality, years of life lost due to premature mortality (YLLs), years lived with disability (YLDs) and disability-adjusted life years (DALYs) for household air pollution from solid cookfuel use (HAP) in Iran, 1990-2013 were estimated based on the Global Burden of Disease Study 2013 (GBD 2013). The burden of disease attributable to HAP was quantified by the comparative risk assessment method using four inputs: (1) exposure to HAP, (2) the theoretical minimum risk exposure level (TMREL), (3) exposure-response relationships of related causes (4) disease burden of related causes. All across the country, solid fuel use decreased from $5.26 \%$ in 1990 to $0.15 \%$ in 2013 . The drastic reduction of solid fuel use leaded to DALYs attributable to HAP fell by $97.8 \%$ (95\% uncertainty interval 97.7-98.0\%) from 87,433 (51072-144303) in 1990 to 1889 (1016-3247) in 2013. Proportion of YLLs in DALYs from HAP decreased from $95.7 \%$ in 1990 to $86.6 \%$ in 2013. Contribution of causes in the attributable DALYs was variable during the study period and in 2013 was in the following order: ischemic heart disease for $43.4 \%$, chronic obstructive pulmonary disease for $24.7 \%$, hemorrhagic stroke for $9.7 \%$, lower respiratory infections for $9.3 \%$, ischemic stroke for $7.8 \%$, lung cancer for $3.4 \%$ and cataract for $1.8 \%$. Based on the Gini coefficient, the spatial inequality of the disease burden from HAP increased during the study period. The remained burden of disease was relatively scarce and it mainly occurred in seven southern provinces. Further reduction of the disease burden from HAP as well as compensation of the increasing spatial inequality in Iran could be attained through an especial plan for providing cleaner fuels in the southern provinces.
\end{abstract}

\section{Introduction}

Globally, the most important cause of household air pollution is solid fuel use. More than three billion people mainly lived in rural and poor urban areas of low- and middle-income countries use solid fuels, including biomass (wood, dung, crop residues and charcoal) and coal for cooking, heating, boiling water and lighting. Incomplete combustion of solid fuels in open fires or inefficient stoves often under poorly ventilated conditions results in emission of large amounts of air pollutants affecting both outdoor and especially household air quality that can lead to a number of adverse health effects (Balakrishnan et al., 2013; Chafe et al., 2014; Clark et al., 2013; WHO, 2015). In addition to air pollution, household solid fuel use is also linked to some other public health, welfare, social, and environmental problems such as injury and violence during fuel collection, time wasting, deforestation, and climate change through emission of carbon dioxide, methane, black

\footnotetext{
* Corresponding author.

E-mail address: r.saeedi@sbmu.ac.ir (R. Saeedi).
} 
carbon, and brown carbon. Due to the multiple impacts, household solid fuel use has been simultaneously considered as an indicator for public health, environmental sustainability, and development at national and international levels (Amegah and Jaakkola, 2016; Bonjour et al., 2013; Gall et al., 2013; WHO, 2015).

The solid fuel use has been included as a risk factor in comparative risk assessment (CRA) of the Global Burden of Disease Study (GBD) from the first attempt in 2004. Although exposure to both household and outdoor air pollution from solid fuel combustion for all the household uses can threaten public health, the estimated burden of disease is attributed to household air pollution from solid cookfuel use (HAP) because currently available exposure data and exposure-response relationships focus only on household air pollution from solid fuel use for cooking (Bonjour et al., 2013; Braubach et al., 2011; Sehgal et al., 2014; Smith et al., 2014).

Epidemiological studies on health outcomes of HAP were started in the 1980s and have continued to the present. By increasing the epidemiological evidences, the health outcomes attributed to HAP have risen from the first GBD CRA in 2004 (including pneumonia, chronic obstructive pulmonary disease (COPD), and lung cancer) to the third one in 2015 (including cataract, COPD, hemorrhagic stroke, ischemic heart disease, ischemic stroke, lower respiratory infections, and lung cancer) (Bonjour et al., 2013; Forouzanfar et al., 2015; Smith et al., 2014; WHO, 2015). Based on the lastest GBD (GBD, 2013, 2016), globally HAP accounted for about 2.9 million deaths and 101.6 million disability-adjusted life years (DALYs) in 1990 and about 2.9 million deaths (almost constant) and 80.1 million DALYs (20\% reduction) in 2013. In ranking level three global risk factors in terms of DALYs, HAP was the seventh among all risk factors and the first among environmental risks in 2013. Although global, regional, and national health losses of risk factors including HAP have been released in the GBD study, national and sub-national study on any risk factor is strictly recommended by the GBD study group to inform national and local decision makers with more detailed and accurate data (Bonjour et al., 2013; Forouzanfar et al., 2015; Murray et al., 2015).

The objective of this research was to estimate national and subnational age-sex specific and cause-specific mortality, years of life lost due to premature mortality (YLLs), years lived with disability (YLDs), and DALYs attributable to HAP in Iran, 1990-2013 based on the GBD (2013). Exposure to HAP was evaluated using national and subnational databases and was then used in the CRA. Spatiotemporal trend of the disease burden attributable to HAP and the most effective points for interventions were also determined.

\section{Materials and methods}

\subsection{The study area}

This study was done in Iran at national, provincial, and rural-urban levels. Iran, a county located in the southwest of Asia, is the eighteenth largest country in the world with an area of $1,648,195 \mathrm{~km}^{2}$. The population of Iran in 2016 is over 79,000,000 and $73.8 \%$ of them (over $58,000,000$ ) live in urban communities. According to the World Bank database, nominal gross domestic product (GDP) per capita based on purchasing power parity of Iran is reported to be 17,366 USD in 2014 that put the country in the middle ranks (The World Bank, 2016).

\subsection{Estimating the burden of disease attributable to HAP}

The burden of disease attributable to HAP was estimated using the CRA approach according to the GBD (2013). In the CRA methodology, the burden disease attributable to past exposure to a risk factor is estimated as a fraction of the disease burden of related cause or causes observed in a given year through comparing observed health outcomes to those that would have been observed if a counterfactual exposure distribution had occurred in the past. The counterfactual exposure distribution of HAP was determined on the assumption that no household uses solid cookfuels. The counterfactual exposure resulted in households annual mean $\mathrm{PM}_{2.5}$ (concentration of particulate matter with aerodynamic diameter smaller than $2.5 \mu \mathrm{m}$ ) of $5.9-8.7 \mu \mathrm{g} / \mathrm{m}^{3}$ as the theoretical minimum risk exposure level (TMREL) that represents roughly the cleanest cities. The disease burden attributable to HAP was computed using the below equation (Ezzati et al., 2004; Forouzanfar et al., 2015; Poursafa et al., 2015; Smith et al., 2014):

$A B_{\text {aspt }}=\sum^{7} D A L Y_{\text {caspt }} \times P A F_{\text {caspt }}$

where $A B_{\text {aspt }}$ is the attributable burden of HAP in age group $a$, sex $s$, province $p$, and year $t, D A L Y_{\text {caspt }}$ is DALYs of cause $c$ (of seven relevant outcomes of HAP) in age group $a$, sex $s$, province $p$, and year $t$ and $P A F_{\text {caspt }}$ is the population attributable fraction (PAF) of cause $c$, age group $a$, sex $s$, province $p$, and year $t$.

$A B_{\text {aspt }}$ was separately calculated for rural and urban communities. In the CRA, the health outcomes attributed to HAP were cataract, COPD, hemorrhagic stroke, ischemic heart disease, ischemic stroke, lower respiratory infections, and lung cancer. Attributable deaths, YLLs, and YLDs were calculated using the same way and equation presented above for DALYs. The $P A F_{\text {caspt }}$ for HAP was computed using the following equation (Ezzati et al., 2004; Forouzanfar et al., 2015):

$P A F_{\text {caspt }}=\frac{\sum_{x=1}^{2}\left(R R_{\text {casp }}(x) \times P_{\text {caspt }}(x)\right)-R R(T M R E L)}{\sum_{x=1}^{2}\left(R R_{\text {casp }}(x) \times P_{\text {caspt }}(x)\right)}$

where $R R_{\text {casp }}(x)$ is the relative risk for exposure level $x$, cause $c$, age group $a$, sex $s$, and province $p, P_{\text {caspt }}(x)$ is the population fraction for exposure level $x$, cause $c$, age group $a$, sex $s$, province $p$, and year $t$ and $R R(T M R E L)$ is the RR of the TMREL that equals to 1 representing no contribution to the disease burden.

In the CRA, two levels of exposure were defined: $\mathrm{PM}_{2.5}$ under solid fuel use for cooking and the TMREL corresponding to no solid fuel use. Personal exposure to $\mathrm{PM}_{2.5}$ under using solid cookfuel for women, men, and children were considered to be 337 (95\% confidence interval (CI) 238-479), 204 (144-290), and 285 (201-405) $\mu \mathrm{g} / \mathrm{m}^{3}$, respectively. The population fraction relying mainly on solid fuels for household cooking was determined from National Population and Housing Census in 1986, 1996, 2006, and 2011 (SCI, 1987, 1997, 2007, 2012). Other national and sub-national household fuel use statistics including number of piped natural gas (PNG) network customers and amount of petroleum fuels sold to household sector (kerosene, gas oil, and liquefied petroleum gas (LPG)) were also used to estimate proportion of household solid fuel use for between years (SCI, 2016). The population data at national and subnational levels including total population and agesex distribution were determined from National Population and Housing Census in 1986, 1996, 2006, and 2011 (SCI, 1987, 1997, 2007, 2012). All the health outcomes and their disease burden and RRs for exposure to HAP were extracted from the GBD, 2013 and database of the Institute for Health Metrics and Evaluation (IHME) (GBD, 2013, 2016; Naghavi et al., 2015). Geographic inequality of the disease burden attributable to HAP was assessed based on the population weighted Gini coefficient, with values ranging from 0 (perfect equality) to 1 (perfect inequality) as explained by Naghavi et al. (2015).

\section{Results and discussion}

\subsection{Household fuel use}

Fig. 1 shows the trend of household fuel use for cooking in Iran from 1990 to 2013. As can be seen in Fig. 1, during 1990-2013 the fraction of population using solid fuels as the main cooking fuel fell by $97.2 \%$, from $5.26 \%$ in 1990 to $0.15 \%$ in 2013 . Because of the substantial improvement, total population exposed to HAP decreased from $2,820,000$ to 113,000 . The remained people using solid cookfuels were mainly villagers and nomads. 


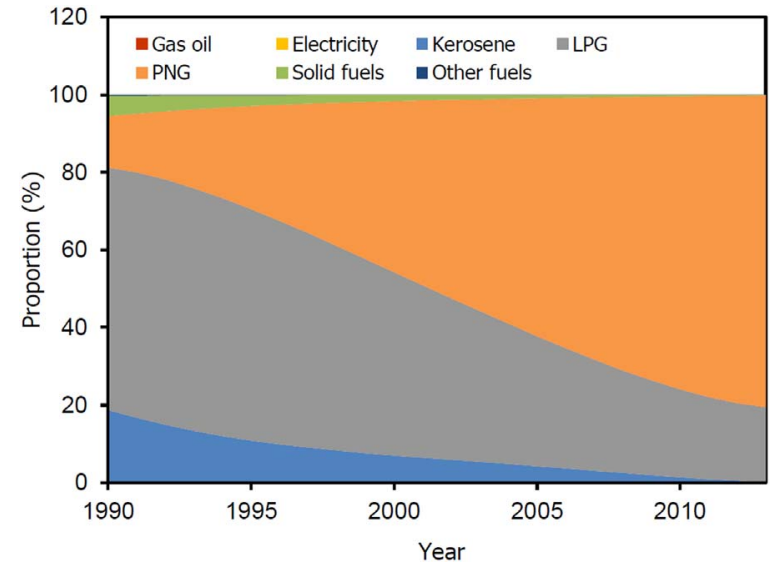

Fig. 1. Trend of household fuel use for cooking in Iran from 1990 to 2013 (LPG: liquefied petroleum gas and PNG: piped natural gas).

The proportions of solid fuel use obtained in this study are lower than those estimated for Iran by Bonjour et al. (2013). During 1980-2010, global use of solid cookfuels decreased from $62 \%$ to $41 \%$, but the number of people using solid cookfuels remained constant at 2.8 billion. Although downward trends in the share of solid cookfuel use were reported for all regions of the world, the curve slopes were relatively low leading to so many people were assessed to use solid cookfuels even in Americas (14\%) and Europe (7\%) in 2010. The observed proportion of solid cookfuel use in Iran for 1990-2013 is lower than the proportion in the region and neighboring countries (Bonjour et al., 2013).

The pattern of household fuel use for cooking was significantly changed toward more use of PNG. The share of household PNG use rose from $13.2 \%$ in 1990 to $80.4 \%$ in 2013 , implying that solid fuels, kerosene, and LPG were substituted by PNG in the most cases. Natural gas combustion also emits air pollutants, but the fuel is relatively clean and its adverse effect on indoor air quality is very lower than solid fuels. The results supported that low price of petroleum fuels and rapid development of PNG network in line with the country's policies of poverty alleviation were successful in reducing solid fuel use and relevant health risks. The proportions higher than $1 \%$ of household solid cookfuel use in 2013 were only observed in rural communities of the provinces Chahar Mahaal and Bakhtiari, Hormozgan, Kerman, Khuzestan, Kohgiluyeh and Buyer Ahmad, Lorestan, and Sistan and Baluchestan. The provinces were the most effective points for an especial plan for providing cleaner fuels to further reduce solid fuel use in Iran.

\subsection{National mortality, YLLs, YLDs, and DALYs}

Table 1 provides deaths and death rate attributable to HAP by cause for rural and urban communities and both sexes in 1990 and 2013 at the national level. All cause deaths attributable to HAP in 1990 and 2013 were estimated to be 2222 (95\% uncertainty interval (UI) 1336-3582) and 83 (48-134), respectively. Ischemic heart disease and lung cancer respectively accounted for the highest and lowest deaths attributable to HAP in both 1990 and 2013 to be 791 (560-1118) and $85(36-163)$ in 1990 and $40(28-55)$ and $3(1-6)$ in 2013. From 1990-2013, deaths and death rate attributable to HAP for any cause fell over 93\% resulting in very low values for 2013. Reduction of HAP death rate (per 100000 people) was slightly higher than that of HAP deaths as a result of population growth. Deaths attributable to HAP were concentrated in rural communities and only less than $3 \%$ of the deaths occurred in urban communities. All cause deaths attributable to HAP for females and males were respectively determined to be 928 (558-1494) and 1295 (778-2089) in 1990 and respectively decreased to $36(20-58)$ and $47(27-76)$ in 2013.
Because of more deaths and death rate from the causes for males, all cause deaths and death rate attributable to HAP for males were higher than those for females during 1990-2013. However, deaths and death rate attributable to HAP from lung cancer and COPD had a different pattern due to much higher RRs for females (Forouzanfar et al., 2015; GBD, 2013, 2016; Naghavi et al., 2015).

National DALYs, DALY rate, and proportion of YLLs in DALYs attributable to HAP by cause for rural and urban communities and both sexes in 1990 and 2013 are given in Table 2. During 1990-2013, all cause DALYs attributable to HAP fell by $97.8 \%$ (97.7-98.0\%) from 87433 (51072-144303) in 1990 to 1889 (1016-3247) in 2013. Contribution of YLLs in all cause DALYs attributable to HAP declined by $9.5 \%(6.9-11.8 \%)$ from $95.7 \%(94.3-97.2 \%)$ in 1990 to $86.6 \%$ (83.1-90.4\%) in 2013. Except for cataract with no mortality (all DALYs accounted for YLDs) and COPD with an YLL proportion of $67.0 \%$ (63.6-70.1\%) in 1990 and $55.2 \%(52.2-58.7 \%)$ in 2013, close to all DALYs attributable to HAP from the other causes were related to mortality (YLDs caused a very low fraction of DALYs). All cause DALYs attributable to HAP for females were 38801 (22452-64536) in 1990 and 843 (433-1472) in 2013. The corresponding values for males were estimated to be 48631 (28620-79767) and 1046 582-1775), respectively. Despite the all cause results that were higher for males, cataract DALYs attributable to HAP occurred only in females and COPD and lung cancer DALYs and DALY rate from HAP were also higher for females because of higher RRs (Forouzanfar et al., 2015; GBD, 2013, 2016; Murray et al., 2015).

All the health metric values attributable to HAP calculated in this study are somewhat different from those estimated for Iran from 1990 to 2013 in the GBD, 2013 (GBD, 2013, 2016). In the GBD, 2013, all cause DALYs and DALY rate attributable to HAP were 68,459 and 122 (lower than this study) in 1990 and 37,643 and 49 (much higher than this study) in 2013, respectively. DALY rate attributable to HAP obtained in this study is much lower than that for North Africa and Middle East, the region that the country is located in, (607 in 1990 and 192 in 2013) and global level (1914 in 1990 and 1132 in 2013), but it is higher than the health metric value for high-income countries $(0.7$ in 1990 and 0.3 in 2013) (Forouzanfar et al., 2015; GBD, 2013, 2016). In contrast with HAP, outdoor air pollution has exhibited an increasing trend and has become an important environmental health problem in Iran. Urban air pollution in large cities such as Tehran, Isfahan, Tabriz, and Shiraz has risen because of increasing road traffic and population growth and in recent years dust storms, mainly originated from southern and western neighboring countries, have caused major concerns and health issues especially in south and west of the country (Ghanbari Ghozikali et al., 2016; Gharehchahi et al., 2013; Gholampour et al., 2014; Janghorbani and Piraei, 2013; Khamutian et al., 2015; Rashki et al., 2012; Shahsavani et al., 2012). Naddafi et al. (2012) estimated that ambient air particulate matter was responsible for a total mortality of 2194 out of 47284 in Tehran in 2010. Based on the GBD, 2013, ambient $\mathrm{PM}_{2.5}$ pollution was the leading risk factor among environmental risks in Iran in 2013 accounting for 430,964 DALYs to be $2.6 \%$ of all DALYs and $37.4 \%$ of DALYs attributable to environmental risks (Forouzanfar et al., 2015; GBD, 2013, 2016).

Fig. 2 shows the trend of national DALYs attributable to HAP by cause from 1990 to 2013. As can be seen in Fig. 2, the total DALYs fell continuously and the greatest rate of decline was caused by lower respiratory infections resulting in the most variable share in the total DALYs. Proportion of causes in national DALYs attributable to HAP during 1990-2013 is presented in Fig. 3. From 1990-2013, cataract accounted for the lowest share to be $0.6-2.4 \%$ of the total DALYs. Contribution of the other causes in the DALYs was as follows: COPD had an increasing trend from $10.6 \%$ in 1990 to $24.7 \%$ in 2013 , proportion of hemorrhagic stroke first rose from $8.8 \%$ in 1990 to $13.3 \%$ in 2000 and then fell to $9.7 \%$ in 2013 , ischemic heart disease had an increasing trend from $23.3 \%$ in 1990 to $43.4 \%$ in 2013 , proportion of ischemic stroke first rose from $6.0 \%$ in 1990 to $9.4 \%$ in 2000 and then fell to 


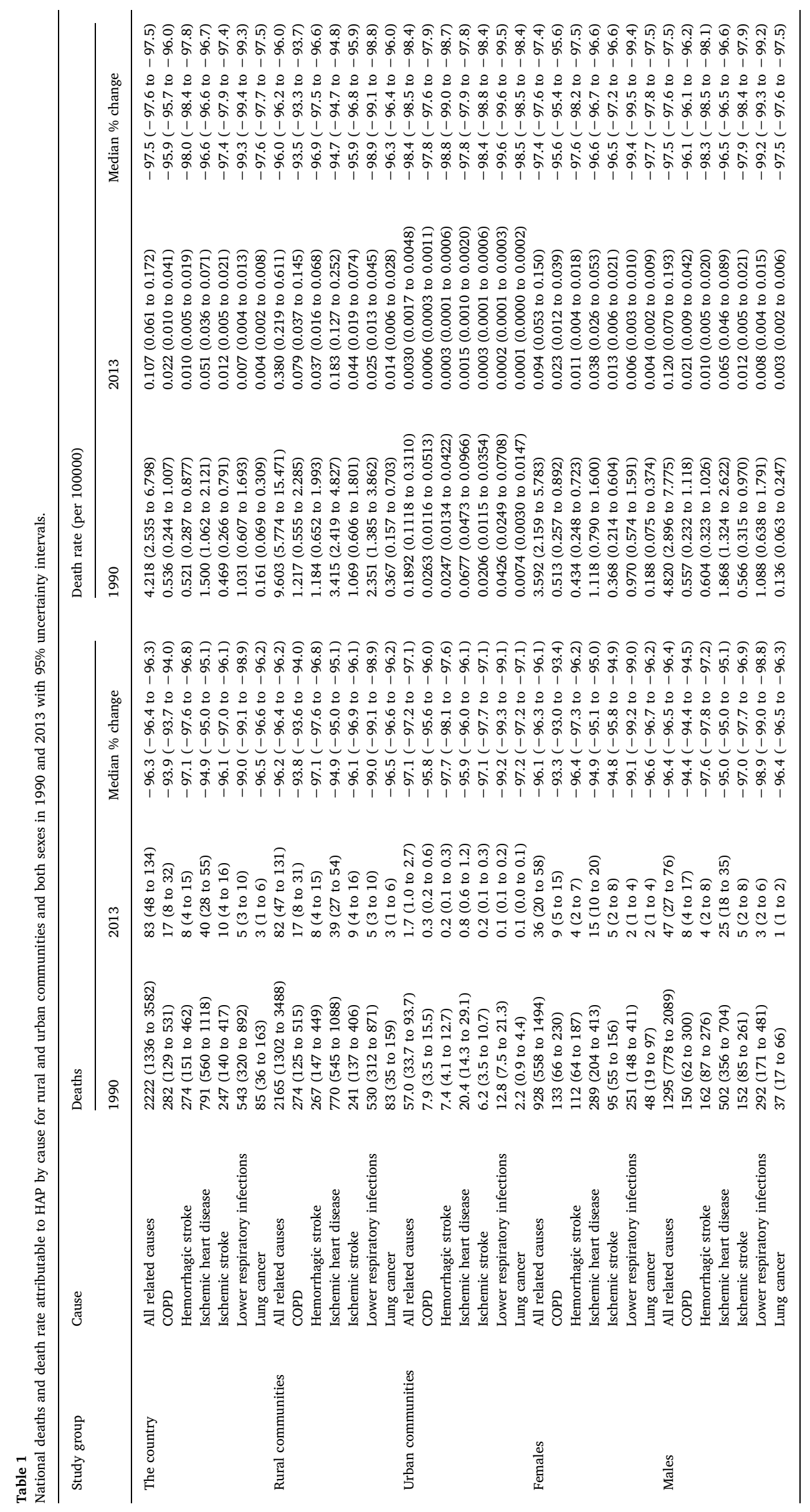




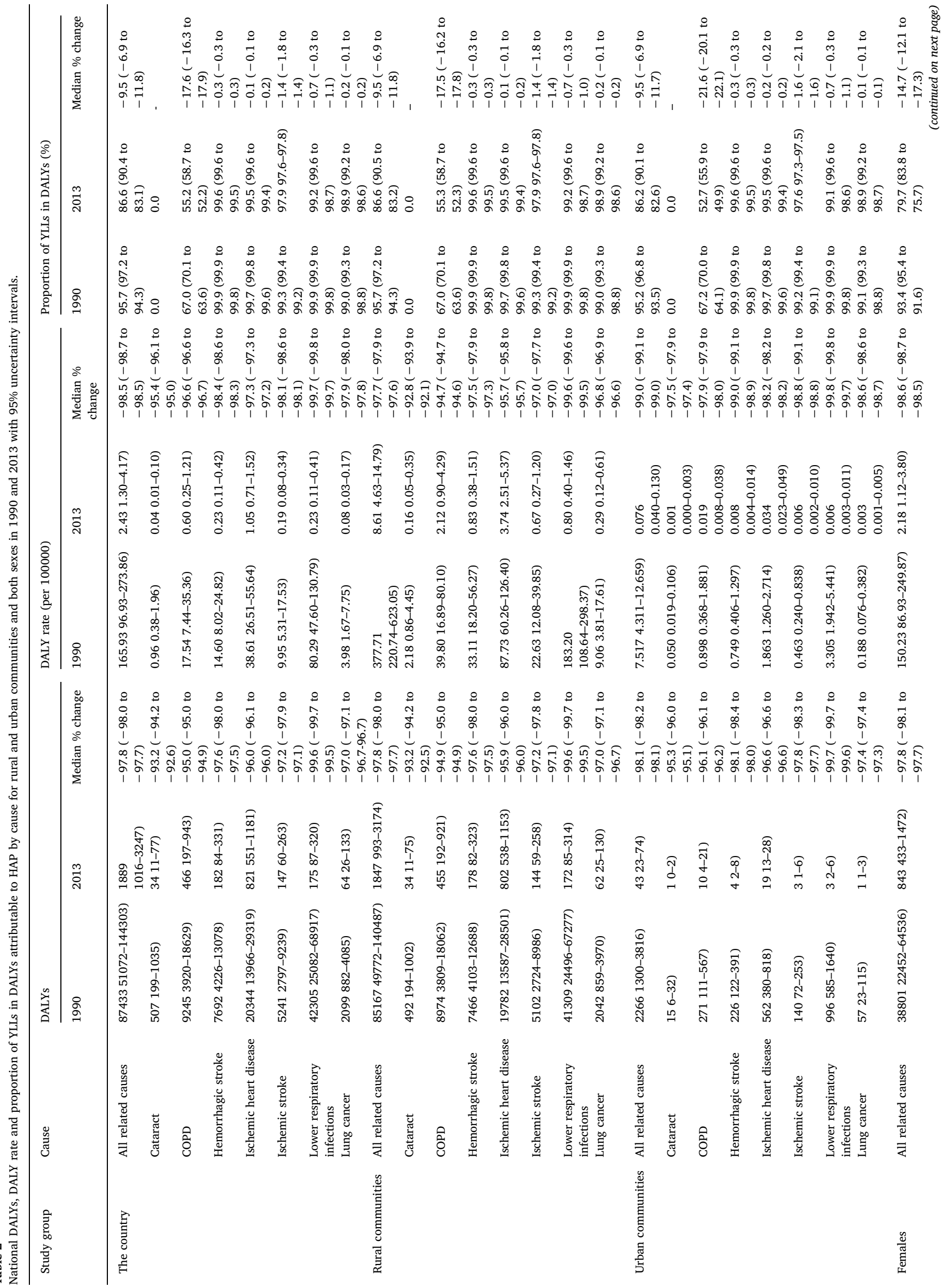




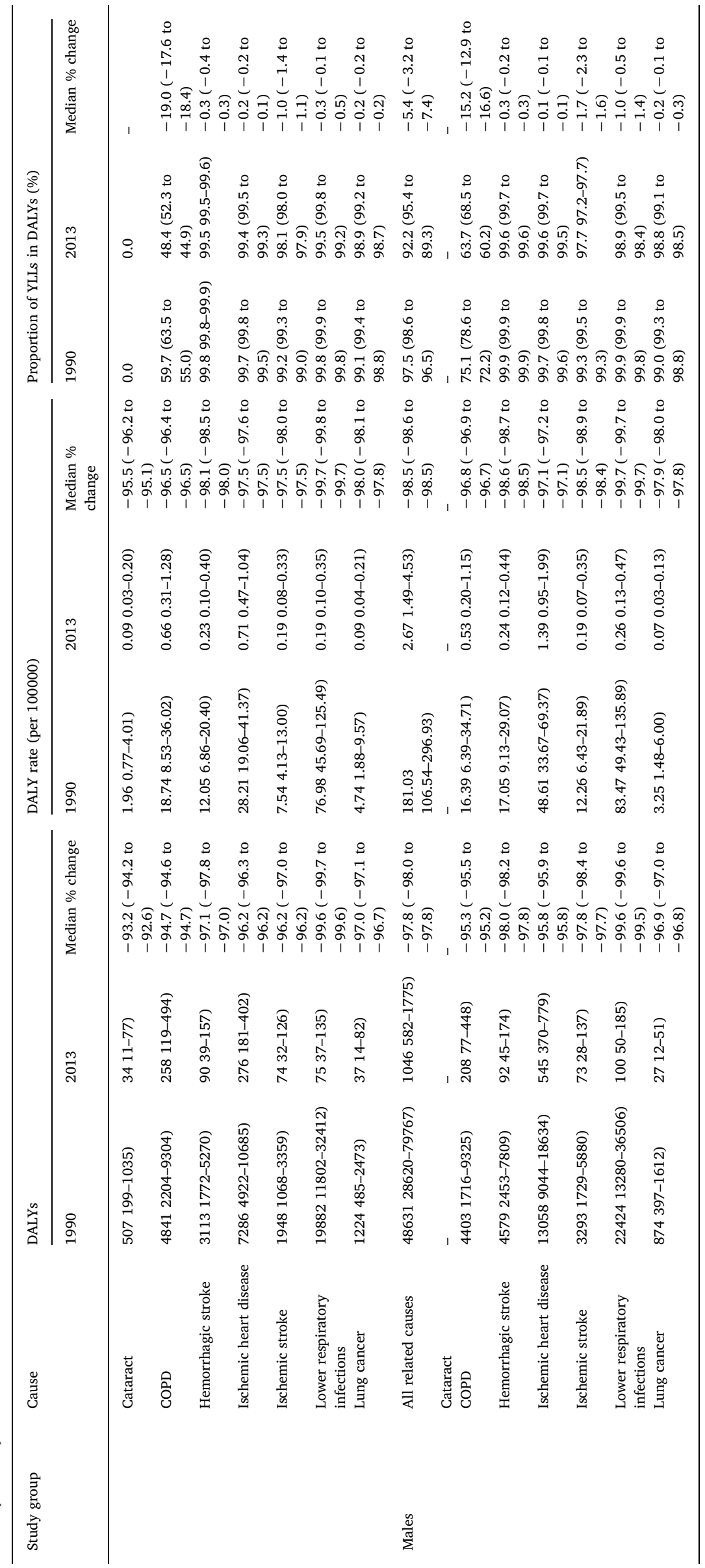




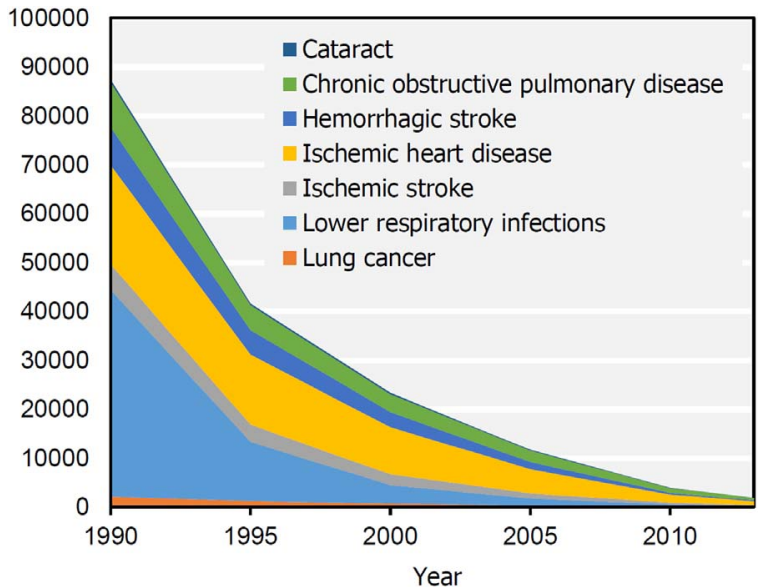

Fig. 2. Trend of national DALYs attributable to HAP by cause from 1990 to 2013.

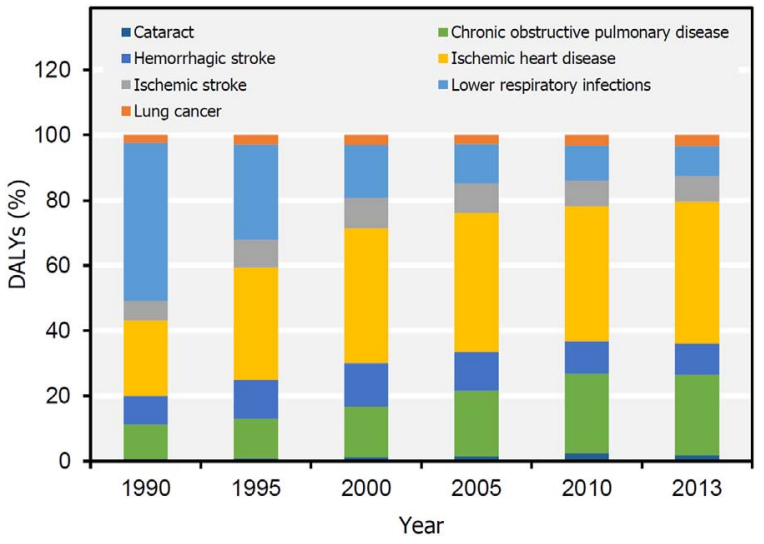

Fig. 3. Proportion of national DALYs attributable to HAP by cause for 1990-2013.

$7.8 \%$ in 2013 , lower respiratory infections had an decreasing trend from $48.4 \%$ in 1990 to $9.3 \%$ in 2013, and lung cancer had an increasing trend from $2.4 \%$ in 1990 to $3.4 \%$ in 2013; therefore, the following order was observed in 2013 on the basis of cause share in DALYs attributable to HAP: ischemic heart disease, COPD, hemorrhagic stroke, lower respiratory infections, ischemic stroke, lung cancer, and cataract.

It should be noted that the estimated burden of disease is not all burden of disease from indoor air pollution because of other health effects of solid fuel use and other sources of indoor air pollution. There are evidences indicating association between household solid fuel use and a number of other health outcomes such as asthma, hypertension, low birth weight, etc. that the causes do not still meet the inclusion criteria of the GBD (Kim et al., 2011; Yan et al., 2016). Indoor air pollution is not only limited to solid fuel use, but other sources including household furnishings, consumer products, and nonsolid fuel (kerosene, LPG, PNG, etc.) combustion can also play an important role especially in middle- and high-income countries with low or no solid fuel use (Lam et al., 2012; Logue et al., 2014; Paulin et al., 2014; Seltenrich, 2014; Smith et al., 2014). Logue et al. (2014) reported that residential natural gas cooking burners significantly increased the indoor concentrations of nitrogen dioxide $\left(\mathrm{NO}_{2}\right)$, carbon monoxide (CO), and formaldehyde (HCHO) even above acute health-based standards, therefore nonsolid fuel use may not be completely safe. More research into the other sources of indoor air pollution and their adverse health effects is needed.

Fig. 4 provides the trend of national PAF for HAP by cause from 1990 to 2013 based on deaths and DALYs. Death PAF for HAP from each cause was higher than its DALY PAF except from cataract indicating HAP contribution to deaths of each cause was higher than the contribution to its DALYs. A substantial decrease was observed in
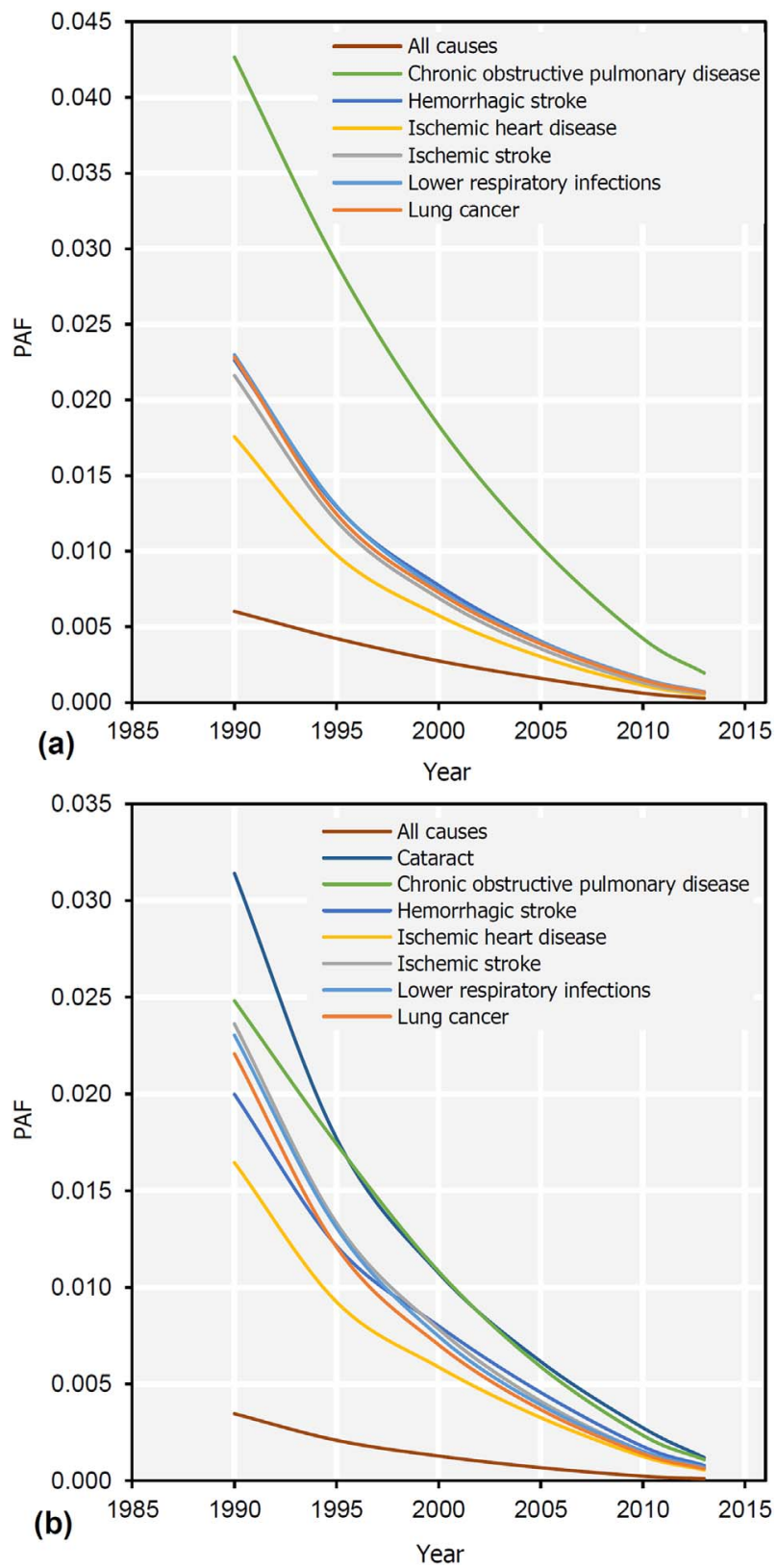

Fig. 4. Trend of national PAF for HAP by cause for 1990-2013 based on: (a) Deaths and (b) DALYs.

the death and DALY PAFs for all of the causes. The risk factor contributions to national all cause deaths and DALYs were respectively estimated to be $6.02 \times 10^{-3}$ and $3.47 \times 10^{-3}$ in 1990 and $2.85 \times 10^{-4}$ and $1.14 \times 10^{-4}$ in 2013 .

Comparing DALY rate attributable to HAP obtained in this study with those of other environmental risks from the GBD (2013) at the national level indicated that HAP declined from ranking fifth (among eight risk factors) in 1990 to the last in 2013; therefore, HAP was not a high rank risk during the study period. According to the GBD (2013) (2016), similar to HAP the other environmental risks at national level accounted for decreased DALY rates in 2013 as compared with 1990 leading to all environmental risk DALY rate of 1489 in 2013 against 3680 in 1990. The GBD (2013) also showed a global shift from environmental risks towards behavioral and metabolic risks during 1990-2013, so that contribution of environmental risks to DALYs fell by $25 \%$ from $15.7 \%$ in 1990 to $11.8 \%$ in 2013 (Forouzanfar et al., 2015). On the other hand, many previous studies showed an increasing trend of environmental contamination all across the world. This discrepancy 

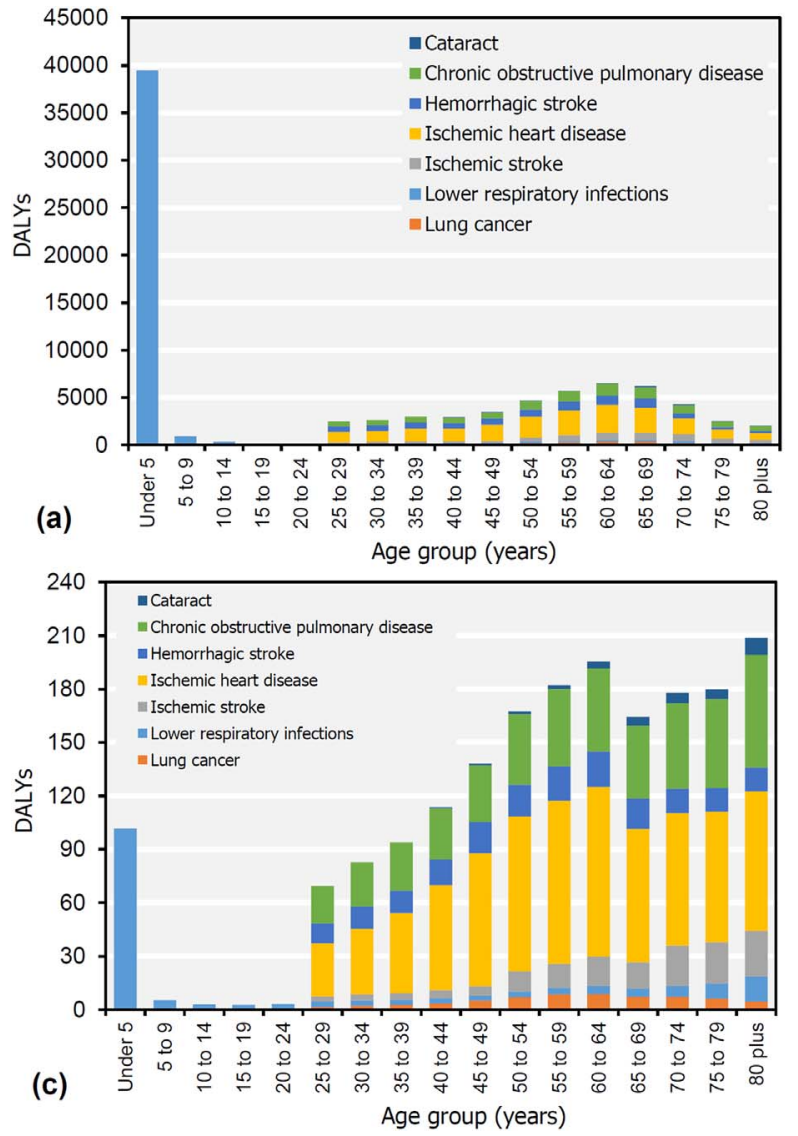

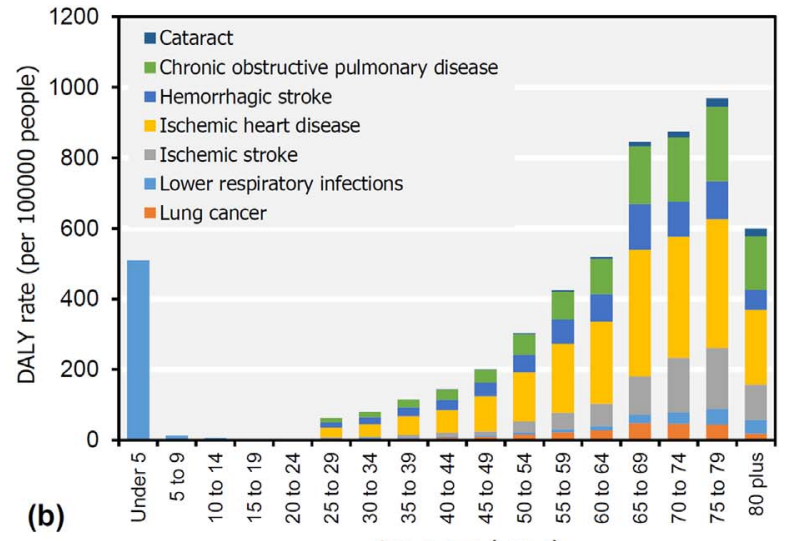

Age group (years)

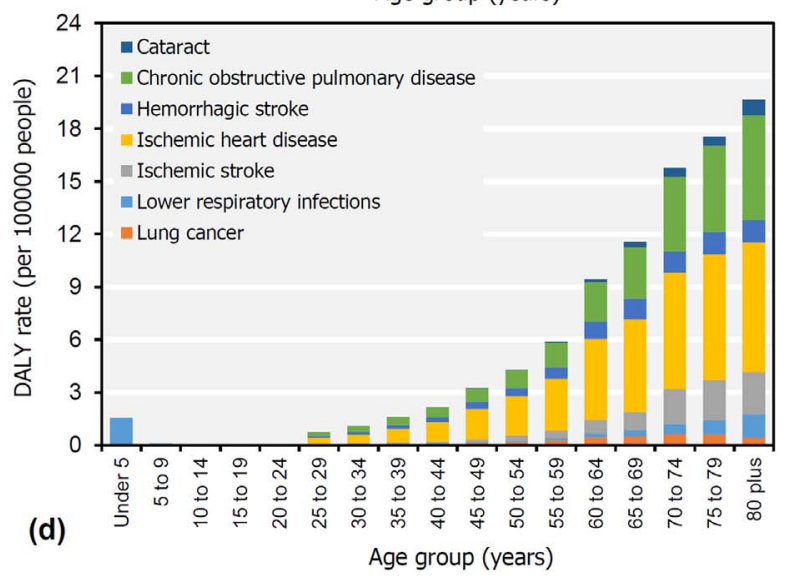

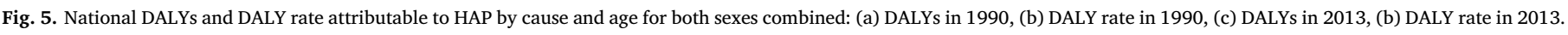

could result from the inclusion of a limited number of environmental risks to the GBD (2013) due to inclusion criteria. The included environmental risks are only the extreme ones causing series problems especially in low-income countries, whereas in middle- and highincome countries many of the risks have been mainly controlled and therefore other environmental risks such as chemical contamination of drinking water and foodstuffs (by pesticides, arsenic, mercury, cadmium, nitrate, fluoride, pharmaceuticals, phenols, phthalates, polychlorinated biphenyls, dioxins, etc.), global warming and climate change, transportation noise, other pollutants of outdoor and indoor air, etc. could be more significant (Bellinger et al., 2016; Boyd and Genuis, 2008; Clark Iii et al., 2012; Ebi and Nealon, 2016; HenríquezHernández et al., 2016; Mohebbi et al., 2013; Vardoulakis et al., 2015; Vienneau et al., 2015; Wells et al., 2011). By inclusion of at least some of these broader causes in future iterations of the GBD, contribution of environmental risks to GBD could be more remarkable and the results would be more accurate.

Fig. 5 displays national DALYs and DALY rate attributable to HAP by cause and age for both sexes combined in 1990 and 2013. According to Fig. 5, the age distribution of the disease burden attributable to HAP was changed during 1990-2013 in a manner that more shares were estimated for older people in 2013. In 1990, the highest DALYs and DALY rate attributable to HAP were observed in the age groups under 5 years and 75-79 years to be 39,495 and 969 , respectively. In 2013, both the health metrics were related to the age group 80 plus to be 209 and 20 , respectively. The total DALYs for the ages under 25 years were only caused by lower respiratory infections. Lower respiratory infections in the age group under 5 years had a considerable contribution (about 45\%) to all DALYs attributable to HAP in 1990 and then the share fell during the study period mainly due to a drastically decrease in the cause DALYs for the age group (GBD, 2013, 2016; Murray et al., 2015).

\subsection{Spatiotemporal trend}

DALY rate attributable to HAP by province and cause in 1990 and 2013 is presented in Fig. 6. The highest DALY rate attributable to HAP was observed in Kohgiluyeh and Buyer Ahmad to be 955 in 1990 (about 6 times higher than the national value) and 22 in 2013 (about 9 times higher than the national value). The other provinces with high DALY rate from HAP in 2013 were respectively Chahar Mahaal and Bakhtiari (13.1), Lorestan (9.6), Khuzestan (8.6), Hormozgan (8.4), Kerman (6.3), and Sistan and Baluchestan (5.6). These provinces are located in southwest, south, and southeast of the country. The areas are also faced with ambient air pollution from dust storms. The simultaneous exposure to indoor and outdoor air pollution can cause synergistic health effects (Ebrahimi et al., 2014; Rashki et al., 2012; Shahsavani et al., 2012).

As indicated in Fig. 6 and based on Gini coefficient (data not shown), inequality in the disease burden attributable to HAP between provinces (and between rural and urban communities) increased during 1990-2013, so that the Gini coefficient for DALY rate attributable to HAP at provincial level rose from 0.50 in 1990 to 0.70 in 2013. Although the decline rate of the disease burden during 1990-2013 is admirable and the remained exposure to HAP is scarce, the increasing spatial disparity is not defendable. In order to further reduce the disease burden attributable to HAP and to compensate the spatial inequality, it is recommended that an especial plan of cleaner fuel supply be designated for the southern provinces with the higher DALY rate.

\section{Conclusions}

National and sub-national burden of disease attributable to HAP in Iran, 1990-2013 were estimated based on the GBD (2013). A 97.2\% decrease of solid cookfuel use during 1990-2013 resulted in a profound 

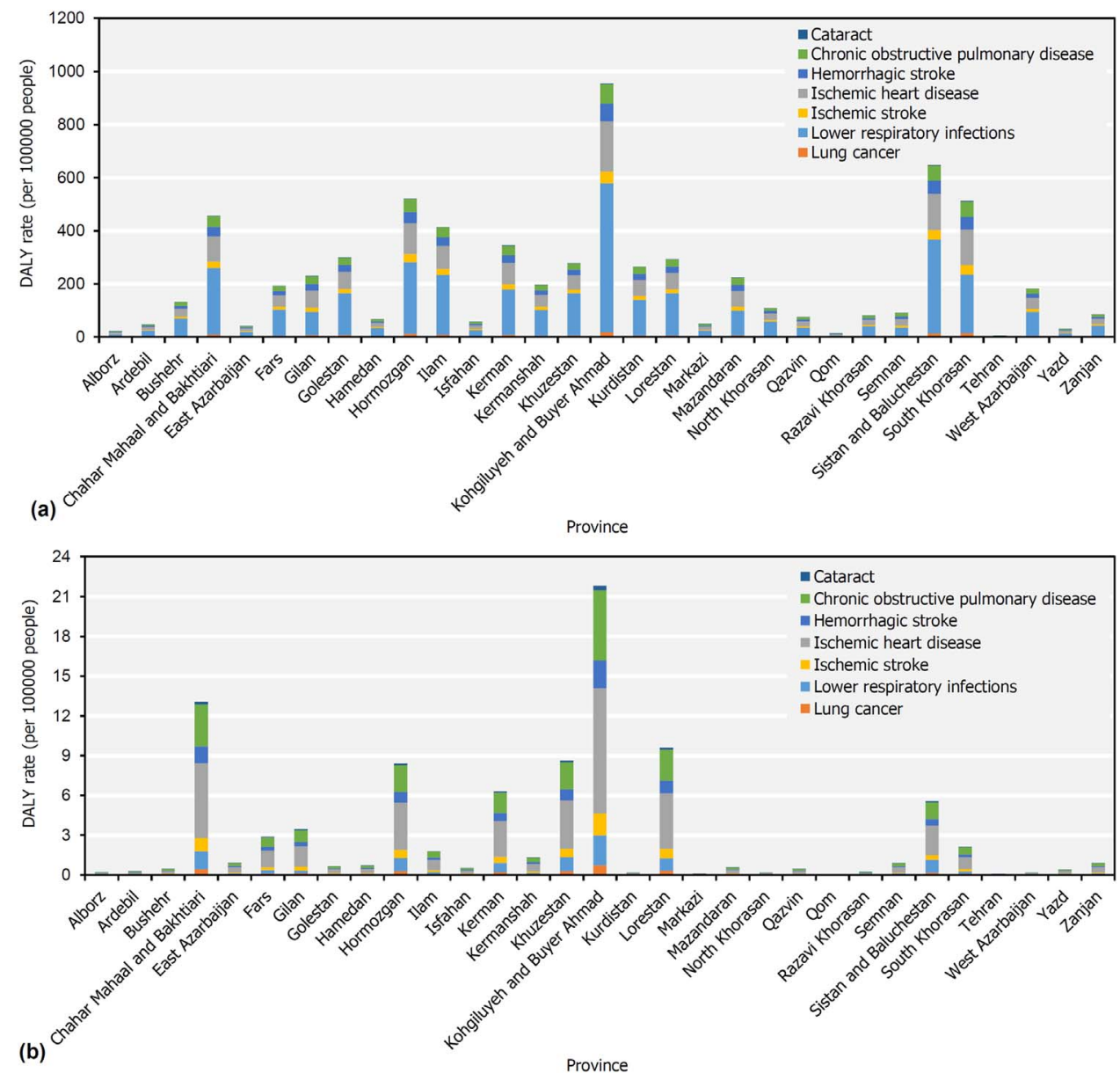

Fig. 6. DALY rate attributable to HAP by province and cause in 1990 (a) and 2013 (b).

decline as high as $98.5 \%$ (98.5-98.7) in DALY rate attributable to HAP from 165.93 (96.93-273.86) in 1990 to $2.43(1.30-4.17)$ in 2013. The rank of HAP among environmental risks decreased from fifth in 1990 to the last in 2013. About $97 \%$ of the attributed burden of disease occurred in rural communities. Most of the disease burden from HAP was caused by mortality, so that YLLs accounted for $95.7 \%$ in 1990 and $86.6 \%$ in 2013 of DALYs attributable to HAP. Contribution of causes in the attributable DALYs in 2013 was in the following order: ischemic heart disease for $43.4 \%$, COPD for $24.7 \%$, hemorrhagic stroke for $9.7 \%$, lower respiratory infections for $9.3 \%$, ischemic stroke for $7.8 \%$, lung cancer for $3.4 \%$, and cataract for $1.8 \%$. From 1990-2013, spatial inequality for DALY rate attributable to HAP increased and most of the remained solid cookfuel use was concentrated in the southern provinces. An especial plan of cleaner fuel supply for the southern provinces could further reduce the disease burden of HAP and redress the increasing spatial inequality in Iran.

\section{Funding sources}

This research was supported by funding from Shahid Beheshti University of Medical Sciences.

\section{Acknowledgments}

The authors thank the staff of Department of Health Sciences, School of Health, Safety and Environment, Shahid Beheshti University of Medical Sciences, Iran, for their collaboration in this research.

\section{References}

Amegah, A.K., Jaakkola, J.J.K., 2016. Household Air Pollution and the Sustainable Development Goals 94. Bulletin of the World Health Organizationpp. 215-221. Balakrishnan, K., Ghosh, S., Ganguli, B., Sambandam, S., Bruce, N., Barnes, D.F., et al., 2013. State and national household concentrations of PM(2.5) from solid cookfuel use: results from measurements and modeling in India for estimation of the global burden of disease. Environ. Health 12 (77-77).

Bellinger, D.C., O'Leary, K., Rainis, H., Gibb, H.J., 2016. Country-specific estimates of the incidence of intellectual disability associated with prenatal exposure to methylmercury. Environ. Res. 147, 159-163.

Bonjour, S., Adair-Rohani, H., Wolf, J., Bruce, N.G., Mehta, S., Prüss-Ustün, A., et al., 2013. Solid fuel use for household cooking: Country and regional estimates for 1980-2010. Environ. Health Perspect. 121, 784-790.

Boyd, D.R., Genuis, S.J., 2008. The environmental burden of disease in Canada: respiratory disease, cardiovascular disease, cancer, and congenital affliction. Environ. Res. 106, 240-249.

Braubach, M., Jacobs, D.E., Ormandy, D., 2011. Environmental Burden of Disease Associated with Inadequate Housing. Copenhagen, Denmark.

Chafe, Z.A., Brauer, M., Klimont, Z., Van Dingenen, R., Mehta, S., Rao, S., et al., 2014. Household cooking with solid fuels contributes to ambient $\mathrm{PM}(2.5)$ air pollution and the burden of disease. Environ. Health Perspect. 122, 1314-1320.

Clark Iii, J.D., Serdar, B., Lee, D.J., Arheart, K., Wilkinson, J.D., Fleming, L. E, 2012 Exposure to polycyclic aromatic hydrocarbons and serum inflammatory markers of cardiovascular disease. Environ. Res. 117, 132-137.

Clark, M.L., Peel, J.L., Balakrishnan, K., Breysse, P.N., Chillrud, S.N., Naeher, L.P., et al., 2013. Health and household air pollution from solid fuel use: the need for improved exposure assessment. Environ. Health Perspect. 121, 1120-1128.

Ebi, K.L., Nealon, J., 2016. Dengue in a changing climate. Environ. Res. 151, 115-123.

Ebrahimi, S.J.A., Ebrahimzadeh, L., Eslami, A., Bidarpoor, F., 2014. Effects of dust storm events on emergency admissions for cardiovascular and respiratory diseases in Sanandaj, Iran. J. Environ. Health Sci. Eng. 12. http://dx.doi.org/10.1186/s40201014-0110-x.

Ezzati, M., Lopez, A.D., Rodgers, A., Murray, C.J.L., 2004. Comparative Quantification of Health Risks: global and Regional Burden of Disease Attributable to Selected Major Risk Factors. World Health Organization, Geneva, Switzerland.

Forouzanfar, M.H., Alexander, L., Anderson, H.R., Bachman, V.F., Biryukov, S., Brauer, M., et al., 2015. Global, regional, and national comparative risk assessment of 79 
behavioural, environmental and occupational, and metabolic risks or clusters of risks in 188 countries, 1990-2013: a systematic analysis for the Global burden of disease study 2013. Lancet 386, 2287-2323.

Gall, E.T., Carter, E.M., Matt Earnest, C., Stephens, B., 2013. Indoor air pollution in developing countries: Research and implementation needs for improvements in global public health. Am. J. Public Health 103, e67-e72.

GBD, 2013. Global Burden of Disease Study 2013 (GBD 2013) Results by Location, Cause, and Risk Factor. Institute for Health Metrics and Evaluation (IHME), Seattle, United States, pp. 2016.

Ghanbari Ghozikali, M., Heibati, B., Naddafi, K., Kloog, I., Oliveri Conti, G., Polosa, R., et al., 2016. Evaluation of chronic obstructive pulmonary disease (COPD) attributed to atmospheric $\mathrm{O}_{3}, \mathrm{NO}_{2}$, and $\mathrm{SO}_{2}$ using Air Q Model (2011-2012 year). Environ. Res. 144, 99-105.

Gharehchahi, E., Mahvi, A.H., Amini, H., Nabizadeh, R., Akhlaghi, A.A., Shamsipour, M., et al., 2013. Health impact assessment of air pollution in Shiraz, Iran: a two-part study. J. Environ. Health Sci. Eng. 11. http://dx.doi.org/10.1186/2052-336X-11-11.

Gholampour, A., Nabizadeh, R., Naseri, S., Yunesian, M., Taghipour, H., Rastkari, N., et al., 2014. Exposure and health impacts of outdoor particulate matter in two urban and industrialized area of Tabriz, Iran. J. Environ. Health Sci. Eng. 12. http://dx.doi. org/10.1186/2052-336X-12-27.

Henríquez-Hernández, L.A., Boada, L.D., Pérez-Arellano, J.L., Carranza, C., Ruiz-Suárez, N., Jaén Sánchez, N., et al., 2016. Relationship of polychlorinated biphenyls (PCBs) with parasitism, iron homeostasis, and other health outcomes: results from a crosssectional study on recently arrived African immigrants. Environ. Res. 150, 549-556.

Janghorbani, M., Piraei, E., 2013. Association between air pollution and preterm birth among neonates born in Isfahan, Iran. J. Res. Med. Sci.: Off. J. Isfahan Univ. Med. Sci. 18, 875-881.

Khamutian, R., Najafi, F., Soltanian, M., Shokoohizadeh, M.J., Poorhaghighat, S., Dargahi, A., et al., 2015. The association between air pollution and weather conditions with increase in the number of admissions of asthmatic patients in emergency wards: a case study in Kermanshah. Med. J. Islam. Repub. Iran. 29 (229229).

Kim, K.-H., Jahan, S.A., Kabir, E., 2011. A review of diseases associated with household air pollution due to the use of biomass fuels. J. Hazard. Mater. 192, 425-431.

Lam, N.L., Smith, K.R., Gauthier, A., Bates, M.N., 2012. Kerosene: a review of household uses and their hazards in low- and middle-income countries. J. Toxicol. Environ. Health Part B, Crit. Rev. 15, 396-432.

Logue, J.M., Klepeis, N.E., Lobscheid, A.B., Singer, B.C., 2014. Pollutant exposures from natural gas cooking burners: a simulation-based assessment for Southern California. Environ. Health Perspect. 122, 43-50.

Mohebbi, M.R., Saeedi, R., Montazeri, A., Azam Vaghefi, K., Labbafi, S., Oktaie, S., et al., 2013. Assessment of water quality in groundwater resources of Iran using a modified drinking water quality index (DWQI). Ecol. Indic. 30, 28-34.

Murray, C.J.L., Barber, R.M., Foreman, K.J., Ozgoren, A.A., Abd-Allah, F., Abera, S.F., et al., 2015. Global, regional, and national disability-adjusted life years (DALYs) for 306 diseases and injuries and healthy life expectancy (HALE) for 188 countries, 1990-2013: quantifying the epidemiological transition. Lancet 386, 2145-2191.

Naddafi, K., Hassanvand, M.S., Yunesian, M., Momeniha, F., Nabizadeh, R., Faridi, S., et al., 2012. Health impact assessment of air pollution in megacity of Tehran, Iran. Iran. J. Environ. Health Sci. Eng. 9. http://dx.doi.org/10.1186/1735-2746-9-28.

Naghavi, M., Wang, H., Lozano, R., Davis, A., Liang, X., Zhou, M., et al., 2015. Global, regional, and national age-sex specific all-cause and cause-specific mortality for 240 causes of death, 1990-2013: a systematic analysis for the Global burden of disease study 2013. Lancet 385, 117-171.

Paulin, L.M., Diette, G.B., Scott, M., McCormack, M.C., Matsui, E.C., Curtin-Brosnan, J., et al., 2014. Home interventions are effective at decreasing indoor nitrogen dioxide concentrations. Indoor Air 24, 416-424.

Poursafa, P., Kelishadi, R., Ghasemian, A., Sharifi, F., Djalalinia, S., Khajavi, A., et al., 2015. Trends in health burden of ambient particulate matter pollution in Iran, 1990-2010: findings from the global burden of disease study 2010. Environ. Sci. Pollut. Res. 22, 18645-18653.

Rashki, A., Kaskaoutis, D.G., Eriksson, P., Qiang, M., Gupta, P., 2012. Dust storms and their horizontal dust loading in the Sistan region, Iran. Aeolian Res. 5, 51-62.

SCI, 1987. National Population and Housing Census in 1986. Statistical Centre of Iran (SCI), Tehran (in Persian).

SCI, 1997. National Population and Housing Census in 1996. Statistical Centre of Iran (SCI) (Tehran) (in Persian).

SCI, 2007. National Population and Housing Census in 2006. Statistical Centre of Iran (SCI), Tehran (in Persian).

SCI, 2012. National Population and Housing Census in 2011. Statistical Centre of Iran (SCI) (Tehran) (in Persian).

SCI, 2016. Statistics by Topic: sections Environment and Energy. Statistical Centre of Iran (SCI), Tehran (Accessed on 2016 Jun 29, Available from) 〈https://www.amar.org.ir/ english/Statistics-by-Topic>.

Sehgal, M., Rizwan, S.A., Krishnan, A., 2014. Disease burden due to biomass cooking-fuelrelated household air pollution among women in India. Glob. Health Action 7. http:// dx.doi.org/10.3402/gha.v7.25326.

Seltenrich, N., 2014. Take care in the kitchen: Avoiding cooking-related pollutants. Environ. Health Perspect. 122, A154-A159.

Shahsavani, A., Naddafi, K., Haghighifard, N.J., Mesdaghinia, A., Yunesian, M., Nabizadeh, R., et al., 2012. Characterization of ionic composition of TSP and $\mathrm{PM}_{10}$ during the Middle Eastern Dust (MED) storms in Ahvaz, Iran. Environ. Monit. Assess. 184, 6683-6692.

Smith, K.R., Bruce, N., Balakrishnan, K., Adair-Rohani, H., Balmes, J., Chafe, Z., et al., 2014. Millions dead: how do we know and what does it mean? Methods used in the comparative risk assessment of household air pollution. Annu. Rev. Public Health 35, 185-206.

The World Bank, 2016. Gross Domestic Product (GDP) per Capita Based on Purchasing Power Parity. Washington, DC, 2016,. Accessed on Jul 9, Available from: 〈http:// data.worldbank.org/indicator/NY.GDP.PCAP.PP.CD >.

Vardoulakis, S., Dimitroulopoulou, C., Thornes, J., Lai, K.-M., Taylor, J., Myers, I., et al., 2015. Impact of climate change on the domestic indoor environment and associated health risks in the UK. Environ. Int. 85, 299-313.

Vienneau, D., Schindler, C., Perez, L., Probst-Hensch, N., Röösli, M., 2015. The relationship between transportation noise exposure and ischemic heart disease: a meta-analysis. Environ. Res. 138, 372-380.

Wells, E.M., Jarrett, J.M., Lin, Y.H., Caldwell, K.L., Hibbeln, J.R., Apelberg, B.J., et al., 2011. Body burdens of mercury, lead, selenium and copper among Baltimore newborns. Environ. Res. 111, 411-417.

WHO, 2015. WHO Guidelines for Indoor Air Quality: household Fuel Combustion. World Health Organization, Geneva, Switzerland.

Yan, Z., Liu, Y., Yin, Q., Qiu, M., 2016. Impact of household solid fuel use on blood pressure and hypertension among adults in China. Air Qual., Atmosphere Health. http://dx.doi.org/10.1007/s11869-016-0395-2. 